\title{
Sharing Renewable Energy in Smart Microgrids
}

\author{
Ting Zhu*, Zhichuan Huang*, Ankur Sharma*, Jikui Su*, David Irwin§, Aditya Mishra ${ }^{\S}$, Daniel \\ Menasche ${ }^{\dagger}$, and Prashant Shenoy \\ *Department of Computer Science, State University of New York \\ ${ }^{\dagger}$ Department of Computer Science, Federal University of Rio de Janeiro \\ ${ }^{\S}$ Department of Computer Science, University of Massachusetts Amherst
}

\begin{abstract}
Renewable energy harvested from the environment is an attractive option for providing green energy to homes. Unfortunately, the intermittent nature of renewable energy results in a mismatch between when these sources generate energy and when homes demand it. This mismatch reduces the efficiency of using harvested energy by either i) requiring batteries to store surplus energy, which typically incurs $\sim 20 \%$ energy conversion losses; or ii) using net metering to transmit surplus energy via the electric grid's AC lines, which severely limits the maximum percentage of possible renewable penetration. In this paper, we propose an alternative structure wherein nearby homes explicitly share energy with each other to balance local energy harvesting and demand in microgrids. We develop a novel energy sharing approach to determine which homes should share energy, and when, to minimize system-wide efficiency losses. We evaluate our approach in simulation using real traces of solar energy harvesting and home consumption data from a deployment in Amherst, MA. We show that our system i) reduces the energy loss on the $\mathrm{AC}$ line by $60 \%$ without requiring large batteries, ii) scales up performance with larger battery capacities, and iii) is robust to changes in microgrid topology.
\end{abstract}

\section{Categories and Subject Descriptors}

J.7 [Computer Applications]: Computers in Other Systems-Command and control

\section{General Terms}

Design, Measurement, Management

\section{Keywords}

Renewable Energy, Energy Sharing, Microgrids, Battery

\section{INTRODUCTION}

To reduce carbon footprint, energy harvesting devices (e.g., solar panels) are becoming increasingly popular in homes nowadays. With the renewable energy generated by solar

Permission to make digital or hard copies of all or part of this work for personal or classroom use is granted without fee provided that copies are not made or distributed for profit or commercial advantage and that copies bear this notice and the full citation on the first page. To copy otherwise, to republish, to post on servers or to redistribute to lists, requires prior specific permission and/or a fee.

ICCPS'13, April 8-11, 2013, Philadelphia, PA, USA. Copyright 2013 ACM 978-1-4503-1996-6/13/04...\$15.00. panels and energy storage units (e.g., batteries), homes can be integrated as a small independent energy community. The power systems employed in such communities are referred to as microgrids. If the harvested energy surpasses the consumption, then microgrids can work independently and autonomously.

However, the amount of renewable energy harvested normally does not match the amount of energy consumed in individual homes. One solution is to sell the surplus energy to a utility company and get energy from the utility company during energy shortage. However, a large amount of energy generated by homes can destabilize the power system if transmitted through an alternating current (AC) line. Another solution is to store the energy in local batteries. However, this approach requires extremely large size batteries to store energy for a whole day's energy consumption. In this paper, we solve the mismatch of energy harvesting and consumption by introducing energy sharing among homes. If renewable energy can be shared and consumed among homes, the traditional power system will not be interrupted by the renewable energy. To realize the energy sharing, we propose a hybrid design of energy transmission system, which contains both the traditional AC line and a small community level direct current (DC) power line. Compared with traditional AC line, our DC line is significantly shorter. Therefore, the energy transmission loss over DC line is much lower than the loss over the traditional AC line. With energy sharing, the total amount of energy needed from the AC line is significantly reduced, which in turn dramatically reduces the total energy transmission loss from the $\mathrm{AC}$ line.

One of our aims is to keep the system compatible with the traditional AC power grid and reduce the deployment and maintenance cost. Therefore, homes are connected to a single DC power line (called DC main bus). This introduces another design challenge - how to monitor and quantify the amount of energy shared among homes. If homes provide (or extract) energy to (or from) the DC power line at the same time, we cannot fully control the amount of energy shared between different pairs of homes. To address this challenge, we propose a new approach, which is similar to the TDMA (Time Division Medium Access) method used in wireless communications. In our approach, different pairs of homes share energy within different time slots. Our approach enables i) multiple homes to simultaneously provide energy to a single home, and ii) a single home to simultaneously provide energy to multiple homes. In this way, our 
approach not only allows us to monitor and quantify the amount of energy shared among homes but also reduces the time required for energy sharing.

Because transmission loss among homes differs for every pair of homes, an efficient algorithm is needed to control the energy sharing flow. Generally, the energy loss between homes includes two parts: i) energy loss over wires, and ii) energy conversion loss during the battery charging and discharging stages. Energy loss over wires is mainly determined by the distance of homes. To reduce the energy transmission loss, we propose a greedy energy matching algorithm to reduce the distance of energy transmission and the amount of energy needed to be stored in the battery. Specifically, the main contributions of the paper can be summarized as follows:

- To the best of our knowledge, this is the first in-depth work to investigate the co-existence of traditional AC power grid and DC power line for sharing renewable energy among homes.

- We have i) designed an efficient energy sharing system to share energy among homes, and ii) developed a lightweight energy matching algorithm and a practical energy transmission scheduling algorithm to minimize the total energy loss based on the predicted energy consumption and energy harvesting.

- We set up a series of experiments with empirical data to verify the effectiveness of our system. The results indicate that our system can reduce $60 \%$ of energy loss from the $\mathrm{AC}$ line by efficiently sharing energy among homes.

The paper is organized as follows. In Section 2, we discuss the need for energy sharing. Section 3 gives an overview of the system architecture. Models, detailed system design, and evaluation are presented in Sections 4, 5, and 6, respectively. Practical issues and related work are discussed in Sections 7 and 8, respectively. Finally, we conclude our paper in Section 9.

\section{MOTIVATION}

This work is motivated by the mismatch between energy harvesting and energy consumption in a single home as shown in Figure 1. The mismatch occurs mainly due to the mismatch between the time when renewable energy is harvested and each home's peak demand time. As a result, today's Distributed Generation (DG) deployments rely heavily on net metering, where consumers sell the unused energy they produce back to the utility company, which offsets their cost relative to grid energy. DG is a much less attractive option if net metering is not available. Net metering laws and regulations vary widely across states; it is not available in at least four states of United States and the regulations are weak in many others [5]. Unfortunately, even where available, states typically place caps on both the total number of participating customers and/or the total amount of energy contributed per customer [2]. After exceeding these caps, utilities are no longer obligated to accept excess power from DG deployments. For example, the state of Washington caps the total number of participating customers at $0.25 \%$

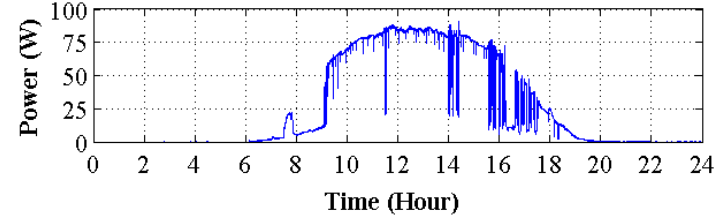

(a) Energy harvesting rate over one day

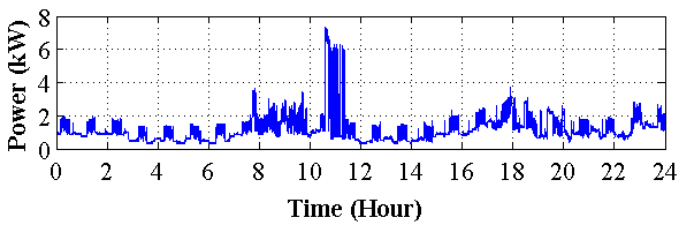

(b) Energy consumption rate over one day

Figure 1: Mismatching between energy harvesting and energy consumption

of all customers. One reason for the strict laws limiting DG's contribution is that injecting significant quantities of power into the grid from unpredictable energy source on a large scale has the potential to destabilize the grid by making it difficult, or impossible, for utilities to balance supply and demand.

Today's energy prices do not make DG financially attractive enough for consumers to reach even these low state caps. However, more widespread adoption of renewable energy sources is critical to meet existing goals. For example, the Renewables Portfolio Standard targets $25 \%$ of electricity generation from intermittent renewables in California [4], while California's Executive Order S-21-09 calls for 33\% of generation from renewables by 2020 [3]. Given current laws, if DG becomes more widespread, residential consumers will have to look beyond net metering to reduce costs and balance on-site energy production and consumption. One alternative approach is to use on-site energy storage, such as batteries. However, batteries are expensive. For example, according to Mckinsey's report, the average price of electric vehicle battery per $\mathrm{kWh}$ is around $\$ 500$ in June 2012 [1]. In [25], we have demonstrated that a $12 \mathrm{kWh}$ battery cannot buffer sufficient renewable energy for a regular home's daily energy consumption, which means we may need every home to be equipped with battery at a price of more than $\$ 6,000$. Moreover, charging and discharging batteries introduce significant energy loss. Therefore, we propose to share harvested energy among small-scale networked homes to balance the energy harvesting and consumption in the microgrid. Because the distances between homes are typically much shorter than the distances from homes to the utility company, energy sharing can significantly reduce energy loss.

\section{SYSTEM OVERVIEW}

In this section, we briefly overview the system architecture and introduce the hardware architecture to achieve energy sharing among multiple homes.

Our energy sharing system uses a cluster controller to control the energy sharing among homes. The cluster controller collects information from homes and then arranges 


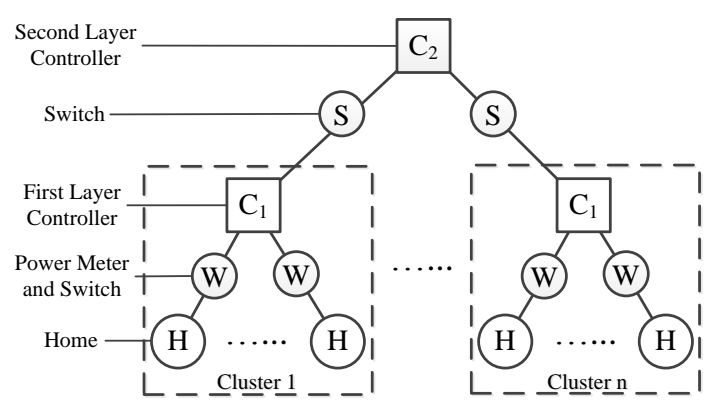

Figure 2: Tree topology of homes

the transmission among homes. To guarantee the backward compatibility with traditional power system, we adopt the tree topology (shown in Figure 2) similar to the one in a traditional power grid to connect homes for energy sharing. Homes are connected to the cluster controller with both a power meter and a switch. The power meter is used to measure energy harvesting and consumption information while the switch is used to control how to share energy with other homes. Because cluster controllers do not need to measure energy information, they are connected to higher layer controllers only through switches. In our system, energy is first shared among homes within a cluster and then shared among homes in different clusters in a higher layer repeatedly until all possible energy transmissions are finished.

To realize energy sharing, we propose a three-layer design, which contains hardware layer, local control layer and global control layer (shown in Figure 3).

- The hardware layer uses a power meter to measure i) energy harvesting rate from renewable energy devices such as solar panels; and ii) energy consumption rate from appliances such as heating devices. This energy information will be periodically transferred to local control layer for prediction of future energy harvesting and consumption rate. The hardware layer will then execute energy transmission with the control signal transferred from power control at the local control layer. The proposed hardware architecture is shown in Figure 4. The harvesting devices (solar panel in our paper) are directionally connected to a power meter and switch because the energy cannot be transmitted to solar panels. Meanwhile, home appliances and battery are bidirectionally connected to a power meter and switch to measure and control the energy flow. The power meter and switch will be connected to DC main bus for energy sharing with other homes.

- The local control layer predicts i) renewable energy that a home expects to harvest based on weather forecast; and ii) expected energy consumption based on historical usage data. Then the amount of energy harvested is compared to the amount of energy consumed for dividing homes into two categories. A home with energy surplus is called an energy supplier while a home with energy shortage is called an energy demander.

- The global control layer uses predicted energy information to determine pair sets of homes for energy shar-

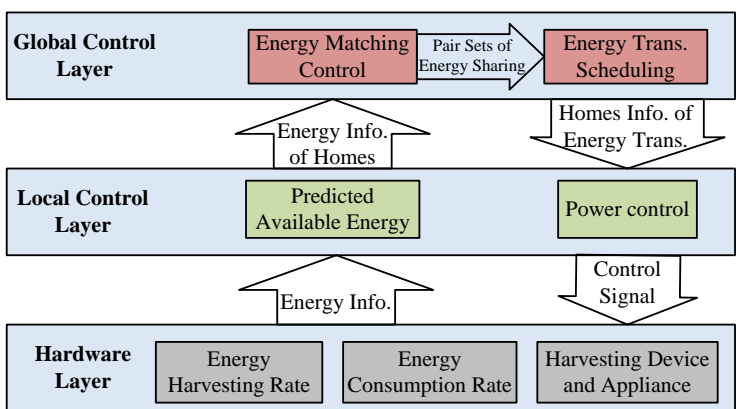

Figure 3: Overview of system architecture

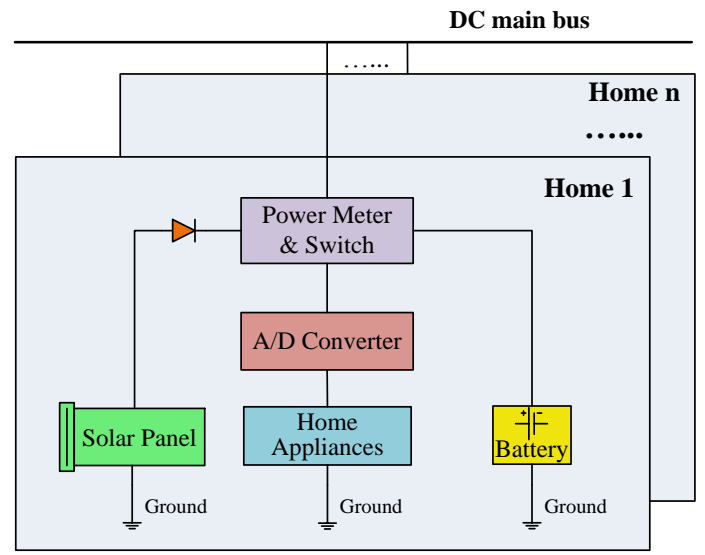

Figure 4: Hardware architecture for energy sharing among multiple homes

ing. Based on the current battery level and the expected available energy, it also determines the amount of energy for a home to share with other homes and to store in its local battery. Then an energy transmission scheduling component decides the order (i.e., schedule) of energy transmission based on matching results. The energy sharing algorithm used in our paper is referred to as Global Energy Sharing (GES). Homes' energy sharing information is transferred to a power controller in the local control layer, and finally control signals would be delivered to hardware for physical energy transmission.

\section{MODEL}

In this section we describe the model used in our paper. Let $X=\{1,2, \ldots, x\}$ be the set of homes. Time is divided into time slots and the size of a slot is referred to as window size $w$. Let $\triangle E_{i}(n w)$ be the difference between harvested energy $E H_{i}(n w)$ and consumed energy $E C_{i}(n w)$ for home $i$ in the time interval $[n w,(n+1) w]$. Homes can be divided into energy suppliers set $S$ and energy demanders set $D$ according to whether $\triangle E_{i}(n w)$ is positive or negative. $S$ and $D$ partition $X$, i.e., $X=S \cup D$ and $S \cap D=\emptyset$.

When $i$ transmits $E_{i \rightarrow j}(n w)$ units of energy to $j$ via DC lines in time interval $[n w,(n+1) w], j$ receives only a fraction of $E_{i \rightarrow j}(n w)$ due to energy loss. The energy loss during transmission contains both energy transmission loss over wires and battery conversion loss. Energy transmission loss over 


\begin{tabular}{|l|l|}
\hline Parameter & Definition \\
\hline$E H_{i}(n w)$ & Harvested energy of $i$ in $[n w,(n+1) w]$ \\
\hline$E C_{i}(n w)$ & Consumption energy of $i$ in $[n w,(n+1) w]$ \\
\hline$\triangle E_{i}(n w)$ & $E H_{i}(n w)-E C_{i}(n w)$ \\
\hline$E_{i \rightarrow j}(n w)$ & Energy transferred from $i$ to $j$ in $[n w,(n+1) w]$ \\
\hline$R_{i \rightarrow j}(n w)$ & Energy loss rate from $i$ to $j$ in $[n w,(n+1) w]$ \\
\hline$E_{i}(n w)$ & Energy lack of $i$ after sharing in $[n w,(n+1) w]$ \\
\hline$R_{i}$ & Energy loss rate of $i$ to get energy from AC line \\
\hline
\end{tabular}

Table 1: Definition of parameters

wires is mainly determined by the amount of energy transmitted, the length and type of power lines and the transmission voltage. During the energy transmission, since renewable energy may not be consumed by a home immediately, extra energy might be stored in a local battery. The portion of energy stored in battery during transmission introduces not only energy transmission loss, but also battery conversion loss during battery charging. Thus, we should store the minimum amount of energy in battery to reduce the energy loss.

Since energy transmission takes time, energy sharing should be based on the future energy information. For example, at time $w$, we use the energy harvesting and consumption information in time interval $[2 w, 3 w]$ to determine how energy should be shared instead of energy information in time interval $[w, 2 w]$.

When a home still lacks energy after sharing, it fetches energy from the power grid via an AC line. This situation occurs mainly either at night when harvested energy is not sufficient or the transmission loss rate $R_{i \rightarrow j}(n w)$ between two homes is larger than transmission loss rate $R_{j}$ over the AC line.

Given supplier set $S$ and demander set $D$ over time, the purpose of energy sharing is to generate an ordered pair set $Q_{n}=\left\{\left(S_{1}, D_{1}\right), \ldots,\left(S_{m}, D_{m}\right), \ldots,\left(S_{\left|Q_{n}\right|}, D_{\left|Q_{n}\right|}\right)\right\}$ at time $n w$ to determine energy sharing in time interval $[n w,(n+1) w]$, where $S_{m} \subset S, D_{m} \subset D$ and $\left|Q_{n}\right|$ is the size of $Q_{n}$. Our goal is to minimize the total amount of energy loss from battery, $\mathrm{DC}$, and $\mathrm{AC}$ lines:

$$
\sum_{n}\left(\sum_{i, j}\left(E_{i \rightarrow j}(n w) \cdot R_{i \rightarrow j}(n w)\right)+\sum_{i} E_{i}(n w) \cdot R_{i}\right)
$$

with the following constraint:

$$
\left|S_{m}\right|=1 \text { or }\left|D_{m}\right|=1 \quad m=1,2, \ldots,\left|Q_{n}\right|
$$

where $i \in S ; j \in D ; R_{i}$ is the energy transmission loss rate of home $i$ from the AC line and $E_{i}(t)$ is the amount of energy shortage after the energy sharing. Constraint in Equation 2 ensures that there can be i) more than one supplier if they are providing energy to same demander and ii) more than one demander if they are obtaining energy from the same supplier.

An example of energy sharing is shown in Figure 5. With the bipartite graph of supplier set $S$ and demander set $D$, a matching result is given as home 1 provides energy to 2; 3 and 4 provide energy to 5 ; and 7 provides energy to 6

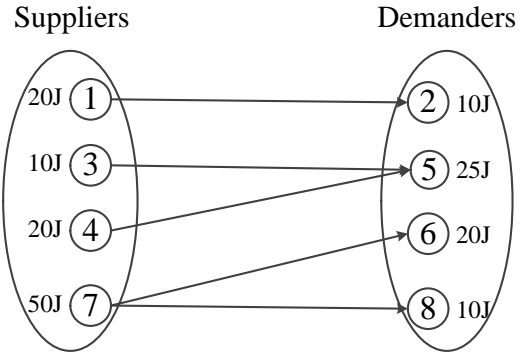

Pair set Q: $\{(\{1\},\{2\}),(\{3,4\},\{5\}),(\{7\},\{6,8\})\}$

Figure 5: An example of matching results $\left(S_{m}\right.$ and $D_{m}$ can be one home or multiple homes. Numbers in $\bigcirc$ is home id and number beside $\bigcirc$ is surplus or shortage energy)

and 8. The energy transmission order follows the order of pair set. Constraint in Equation 2 means we do not allow simultaneous energy sharing such as $(\{1,3\},\{2,5\})$. In this case, we are not able to control whether energy transmission is from 1 to 2 and 3 to 5 or from 1 to 5 and 3 to 2 . The detailed discussion of energy transmission order is described in Section 5.4.

\section{SYSTEM DESIGN}

In this section, we introduce detailed system design for energy sharing. Firstly, we give energy harvesting and consumption prediction algorithms for predicting future energy information. Then, with the predicted energy information, we describe energy sharing within the cluster through matching energy among homes and scheduling energy transmissions. Finally, we explain how the energy sharing can be extended in homes with tree topology.

\subsection{Renewable Energy Prediction}

For renewable energy, we use a prediction model similar to Sharma et al. [24] that translates a weather forecast from the National Weather Service (NWS) into a solar or wind energy harvesting prediction. Since solar energy is the predominant renewable energy source in residential DG deployments, this paper focuses on solar energy. However, the prediction model and energy sharing method can also be applied to other types of renewable energy such as wind energy. We briefly summarize the model below, which uses forecasted sky condition to predict solar energy harvesting. The NWS releases a sky condition forecast, in addition to other weather metrics, every hour for the next 24 hours. At any time $t$, based on predicted sky condition percentage $C(t)$, we compute the solar panel's energy harvesting power $\mathrm{PH}_{i}(t)$ as:

$$
P H_{i}(t)=P_{\max } \cdot(1-C(t))
$$

where $P_{\max }$ is the solar panel's maximum possible harvesting power. Sharma et al. [24] quantify the accuracy of Equation (3) and show that it is more accurate than existing techniques that use the past to predict the future. Thus, based on Equation (3), at any time $t=n w$, we predict the solar energy harvesting within the next energy sharing intervals $w$ as follows: 


$$
\widehat{E H}_{i}((n+1) w)=\int_{n w}^{(n+1) w} P H_{i}(\tau) d \tau
$$

where $w$ is the energy sharing window size.

\subsection{Energy Consumption Prediction}

To predict the home's energy consumption, we use a simple model based on an Exponentially Weighted Moving Average (EWMA). The EWMA exploits the diurnal nature of home consumption, while it also adapts to seasonal variations. On a typical day, we expect the total energy consumption to be similar to the total energy consumption of previous days with slight deviations in weather and daily activities. More sophisticated models that consider changing weekend activity patterns, weather conditions, or other information are possible.

One goal of this work is to quantify how much renewable energy we are able to utilize with a simple and straightforward prediction model. Let $E C_{i}(n w)$ denote the amount of energy consumed in $[n w,(n+1) w]$ and $\widehat{E C}_{i}((n+1) w)$ denote the predicted energy consumed in $[(n+1) w,(n+2) w]$, which is given by:

$$
\widehat{E C}_{i}((n+1) w)=\alpha \cdot \widehat{E C}_{i}(n w)+(1-\alpha) \cdot E C_{i}(n w)
$$

The value of $\alpha$ is chosen by using the method in [13].

\subsection{Energy Matching Algorithm}

With the predicted energy harvesting and consumption information, we introduce the energy matching algorithm in this section. We use a greedy energy search algorithm to match the energy among homes.

The details of matching algorithm are shown in Algorithm 1. With the information of energy harvesting and consumption described in sections 5.1 and 5.2 , we generate a bipartite graph as illustrated in Figure 5. If the harvested energy of home $i$ is larger than its consumed energy, $i$ is categorised as $S$, shown in the left set in Figure 5, otherwise $i$ will be categorised as $D$, shown in the right set in Figure 5 . We sort homes in $D$ in decreasing order based on the amount of energy demanded (Line 1). Then we fetch the first home $j$ in sorted $D$, and match the shortage energy from its nearest neighbor $i$ in $S$ (Lines 2-3). If $i$ has enough energy, we update $E_{i \rightarrow j}(n w)$ and $\triangle E_{i}(n w)$ (Lines 4-5). We also update $(\{*\},\{j\})$ in $Q$ to $(\{*\} \cup\{i\},\{j\})$ and the match of $j$ is finished (Lines 6-8). $\{*\}$ is the matching sets of homes for $j$ in $Q$. Otherwise, we also update the matching result of $j$ in $Q$ and continue to match energy for $j$ until its energy demand is fulfilled (Lines 9-12). After the match of $j$, we continue the match of homes in $D$ repeating the same process (Line $13)$.

Although Algorithm 1 does not guarantee the optimum solution of the energy matching problem, it has a low complexity, which is $|D|(\log |D|+|S| \log |S|)$. As the energy match interval is limited and the number of homes might be large, we adopt Algorithm 1 for energy matching instead of other sophisticated approaches.

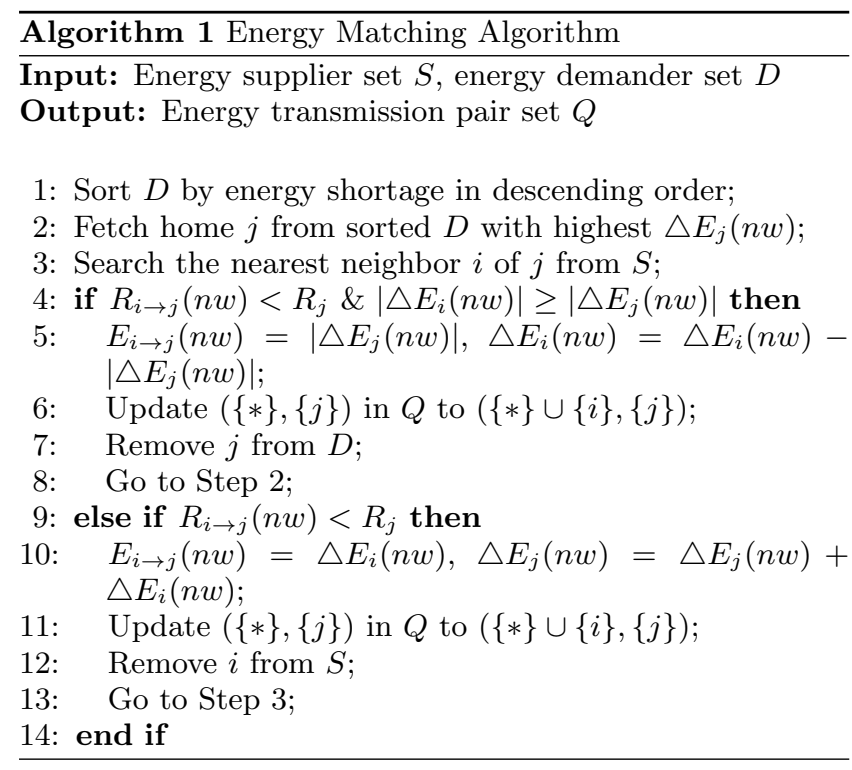

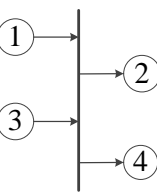

(a) Transmission chaos

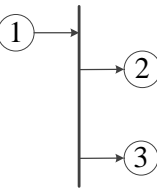

(b) Transmission scheduling
Figure 6: Examples of energy transmission

\subsection{Energy Transmission Scheduling}

With the matching results, we can execute energy transmissions. The homes are all connected to one main bus of DC line shown in Figure 6(a). If two energy transmissions $(\{1\},\{2\})$ and $(\{3\},\{4\})$ occur simultaneously, we cannot ensure the energy is transmitted from home 1 to 2 and 3 to 4 or home 1 to 4 and 3 to 2 , which we refer to as transmission chaos. Because energy transmission pairs are already determined to minimize transmission loss, the transmission from home 1 to 4 and 3 to 2 may cause more energy loss.

To solve the problem, we develop a solution similar to TDMA to schedule energy transmission. The basic idea of transmission scheduling is to have transmissions executed simultaneously only if those transmissions do not cause transmission chaos. Generally, transmissions with one supplier do not cause transmission chaos because demanders cannot fetch energy from other homes and energy transmission flow can be controlled. Transmissions with one demander also do not cause transmission chaos. Thus, our solution is to combine the transmissions that share the same supplier or demander.

According to Algorithm 1, energy matching is processed one by one for homes in $D$. Thus, the transmissions of matching result have only one demander, such as $(\{1\},\{2\})$ or $(\{1,2\},\{3\})$. Our algorithm needs to combine the transmissions with a same supplier. The detailed algorithm is shown in Algorithm 2. Firstly, we combine pair sets such as $(\{1\},\{2\})$ and $(\{1\},\{3\})$ to $(\{1\},\{2,3\})$ to reduce transmission time as shown in Figure 6(b) (Lines 1-5). Since energy matching is based on the energy information of next time 


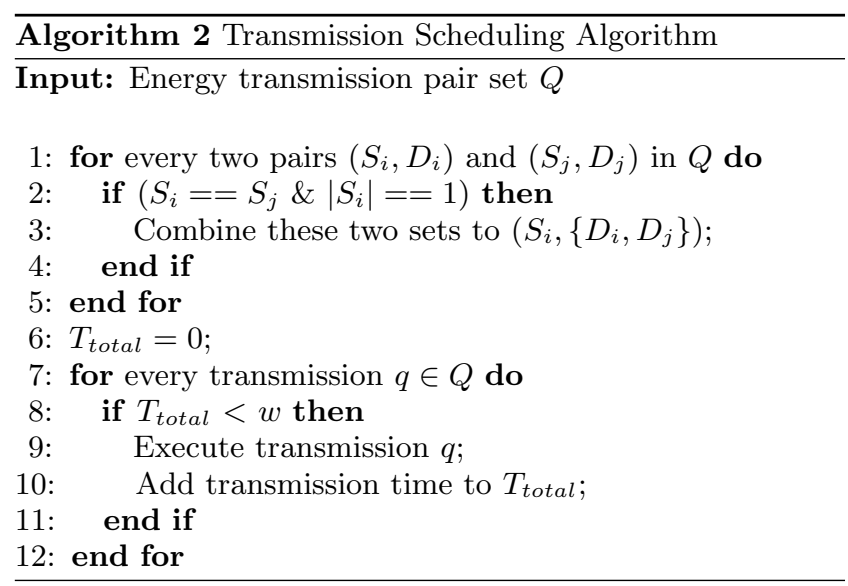

window, total time of energy transmissions should not exceed window size $w$. We initialize total transmission time $T_{\text {total }}=0$ (Line 6$)$. After the combination of transmissions, we execute transmissions sequentially until all the transmissions are finished or total transmission time $T_{\text {total }}$ surpasses $w$ (Lines 7-12).

\subsection{Energy Sharing Among Clusters}

In this paper, we adopt the tree topology in Figure 2 to do energy sharing. This is because i) the current power grid system uses a tree topology, which reduces the cost for reconstruction and design; ii) since only one energy transmission can be executed at a time within the cluster, the number of homes in a cluster should be limited to reduce the energy transmission time. However, the energy matching algorithm and transmission scheduling algorithm (described in Sections 5.3 and 5.4) are mainly applicable for energy sharing within the clusters. In this section, we introduce how these two algorithms can be applied to a system with a tree topology in Algorithm 3.

At the beginning, every cluster will execute Algorithm 1 for homes in that cluster with the energy information obtained from its children (Lines 1-2). The child can be either a home or a cluster of lower layer. Then we initialize the free transmission pair set in cluster $Q^{\prime}$ as $\emptyset$ and total transmission time $T_{\text {total }}$ as 0 (Line 3 ). The cluster checks if there are more than one child of itself free to execute transmission. If yes, it combines the transmissions in those children that can be executed simultaneously (Lines 4-10). A child is free if there is no transmission executing in the child. After combinations, every transmission in each cluster is executed sequentially in a way similar to Algorithm 2 (Lines 11-18).

\section{IMPLEMENTATION AND EVALUATION}

In this section, we evaluate the performance of our energy sharing system. We collect real data of energy harvesting and consumption in Amherst, MA; then we compare efficiency of our system against other solutions; finally we show that our system can work with different battery sizes and home topology.

\subsection{Data Collection}

We collected energy consumption data of 50 homes (shown in Figure 7). We find the peak demand of different homes
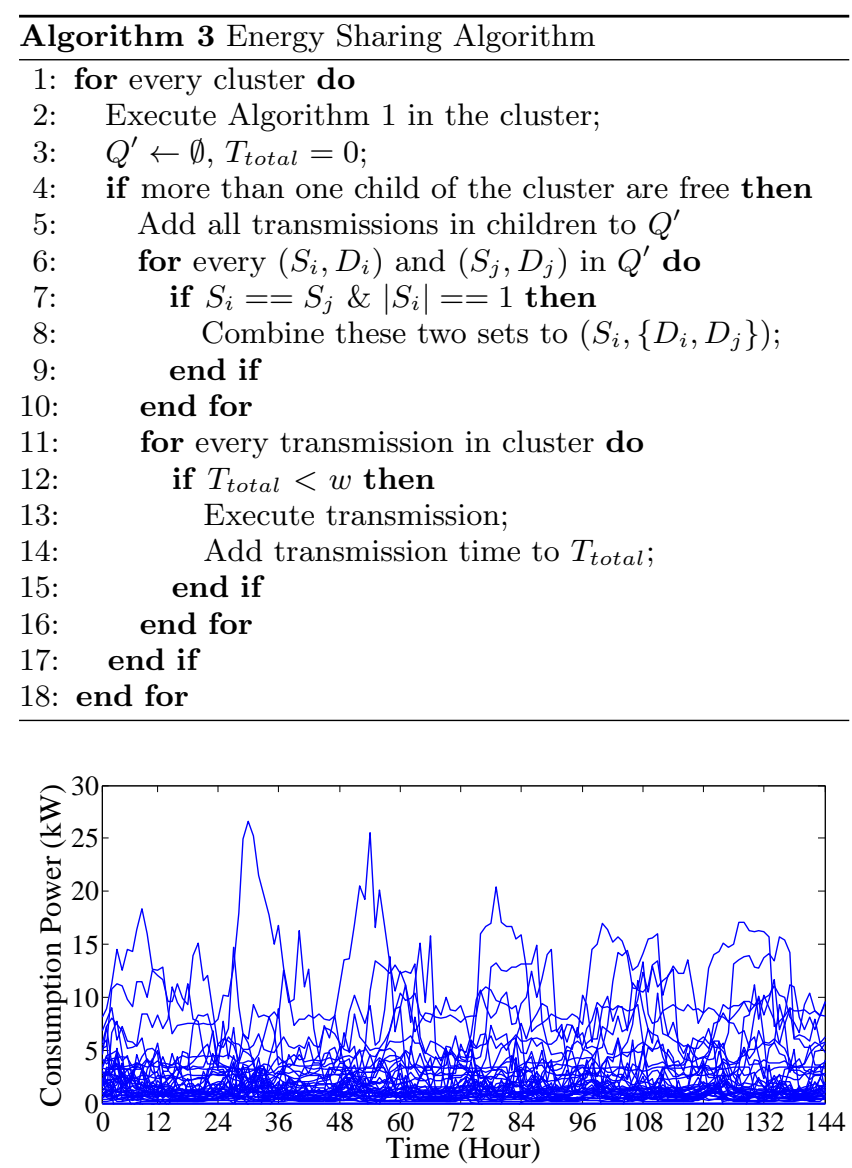

Figure 7: Consumption energy in six days

varies. Furthermore, the peak demand of one home varies on different days. The consumption pattern of homes in our paper are not similar because they are not from the same location. Moreover, even in the same location, people with different living habits and jobs may have different energy consumption patterns.

We also deploy solar panels in one home to collect the energy harvesting data. The energy harvesting of a home is shown in Figure 8. In a day, the solar panel begins to harvest energy at around $7 \mathrm{am}$; it reaches the energy peak around $12 \mathrm{pm}$ and ends the harvesting around $8 \mathrm{pm}$. However, due to the varying weather conditions, the harvesting energy in different days varies. Since the energy harvesting pattern from solar panels is similar in an area, we use the trace to generate energy harvesting data of other homes with some variance.

The energy consumption data of 50 homes is collected every minute over six days. The energy harvesting data is collected every hour over six days. We use the weather forecast data from National Weather Station. Then with the raw data, we calculate the prediction data of energy harvesting and consumption over six days and use it in our simulations.

\subsection{Evaluation Baseline and Metrics}

Baseline: To verify the efficiency of our energy sharing algorithm GES, we compare our design with i) Oracle, which uses the same energy sharing algorithm as GES, but assumes 


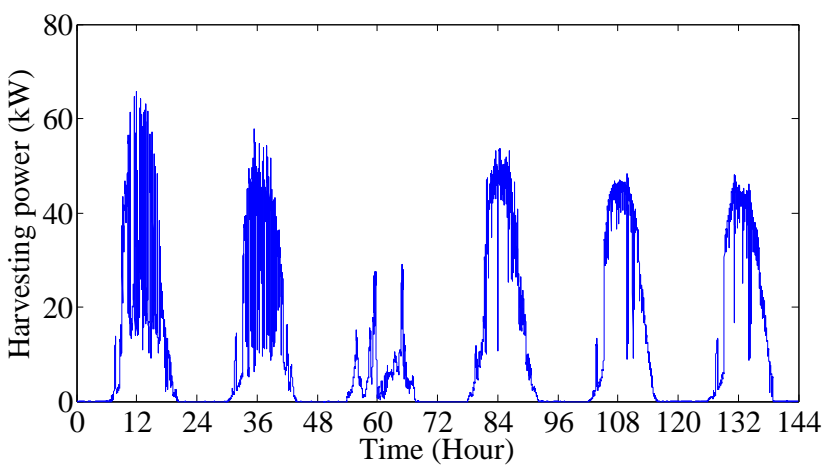

Figure 8: Harvesting energy in six days

real energy consumption and harvesting data for next window is available. In comparison with Oracle, we aim to evaluate the impact of our prediction algorithm; ii) Local Energy Sharing (LES), which only allows homes to share energy with their nearest neighbor, thus the efficiency of energy sharing is low as the constraints for energy sharing are more stringent; iii) No Energy Sharing (NES), which adopts a large battery for every home to store all the surplus harvesting energy for future usage; if there is no energy in battery, it gets energy from AC line.

Metrics: We use two metrics to evaluate the efficiency of our algorithm in our experiment, i) Energy Loss Savings: energy loss of four algorithms compared to the energy loss of homes that always get energy from AC line if local energy does not suffice; ii) Battery Size: the battery size used for homes to store extra energy. One simple way to select battery size for a home is to choose maximum amount of surplus or shortage energy in whole time. However, it will require every home to install large size of batteries. To balance price and energy efficiency, we select battery size for a home to hold maximum amount of surplus or shortage energy in any two consecutive windows.

\subsection{Evaluation Results}

In this section, we evaluate the effectiveness of our energy sharing system, which includes the efficiency of our system, the impact of the window size and the number of homes in a cluster. All results are simulated with the six days empirical data of energy harvesting and consumption introduced in Section 6.1. The battery loss rate we use is $15 \%$ [23]; AC and DC transmission loss rates are around $22.6 \%$ and $7.6 \%$, which varies with different distances of homes [15].

\subsubsection{System Efficiency}

Figure 9 shows the energy loss savings of four different algorithms. In this simulation, the total number of homes varies from 10 to 50 and the number of homes in one cluster is fixed at 10. In all four algorithms, the energy loss savings increase when the total number of homes increases. This is because with more homes, more energy can be shared or stored in local batteries. Our algorithm outperforms LES and is quite close to Oracle. NES performs worst, since the harvesting and consumption in a home usually do not match, thus many homes have less harvesting energy and need to get more energy from $\mathrm{AC}$ line. Other homes that have more harvested energy will need larger batteries to store energy.

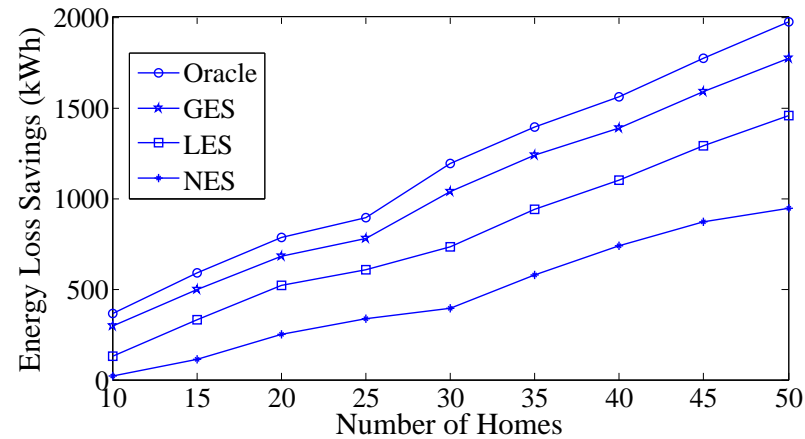

Figure 9: Energy loss savings (Total energy loss of four different algorithms, which includes transmission loss over wires, battery and AC line)

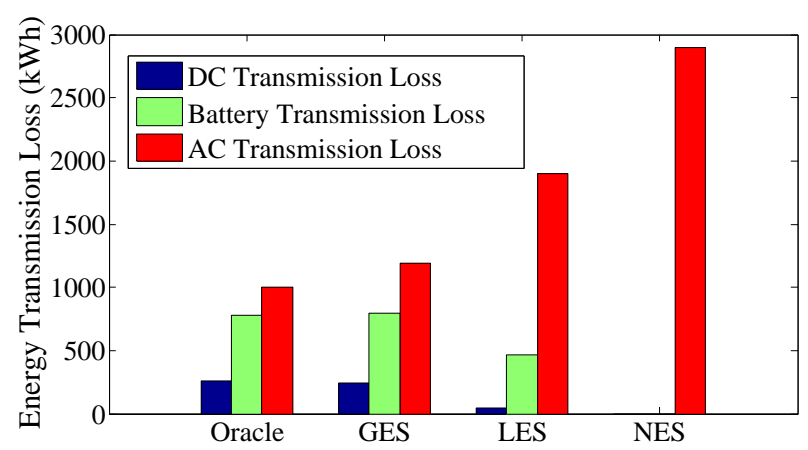

Figure 10: Detailed transmission loss (Transmission loss by DC wires, battery and AC line of 50 homes with 5 clusters)

We also show the detailed transmission loss in Figure 10. The result is for 50 homes of five clusters. The transmission loss over AC line still comprise a large portion of the total energy loss. However, our algorithm reduces $60 \%$ AC transmission loss compared to NES. LES has less energy transmission over DC line than GES, therefore it needs more energy from AC line. Since the transmission loss over AC line is larger than battery conversion loss and DC transmission loss, it explains why transmission loss of LES is larger than GES in Figure 9. Since the predicted data is not accurate, some homes may envision transferring more energy than they can harvest to other homes. In such a case, they have to get energy from AC line after sharing with our algorithm. That explains why our algorithm needs more energy from both AC and DC lines as compared to Oracle.

The battery size needed for every home is shown in Figure 11. Compared to NES, the other three algorithms need smaller size of battery at each home. The distribution of battery size among homes is proportional to the energy harvesting and consumption of homes. Our algorithm and Oracle need almost the same battery size for every home.

The energy transmission over AC line per hour is shown in Figure 12. All three energy sharing algorithms are compared to NES. For Oracle, homes seldom need any energy from AC line except when the harvesting energy from solar panel is not enough in day 3 (Hour 48 to 72). Our algorithm is quite close to Oracle, in which for nearly 10 hours of one 


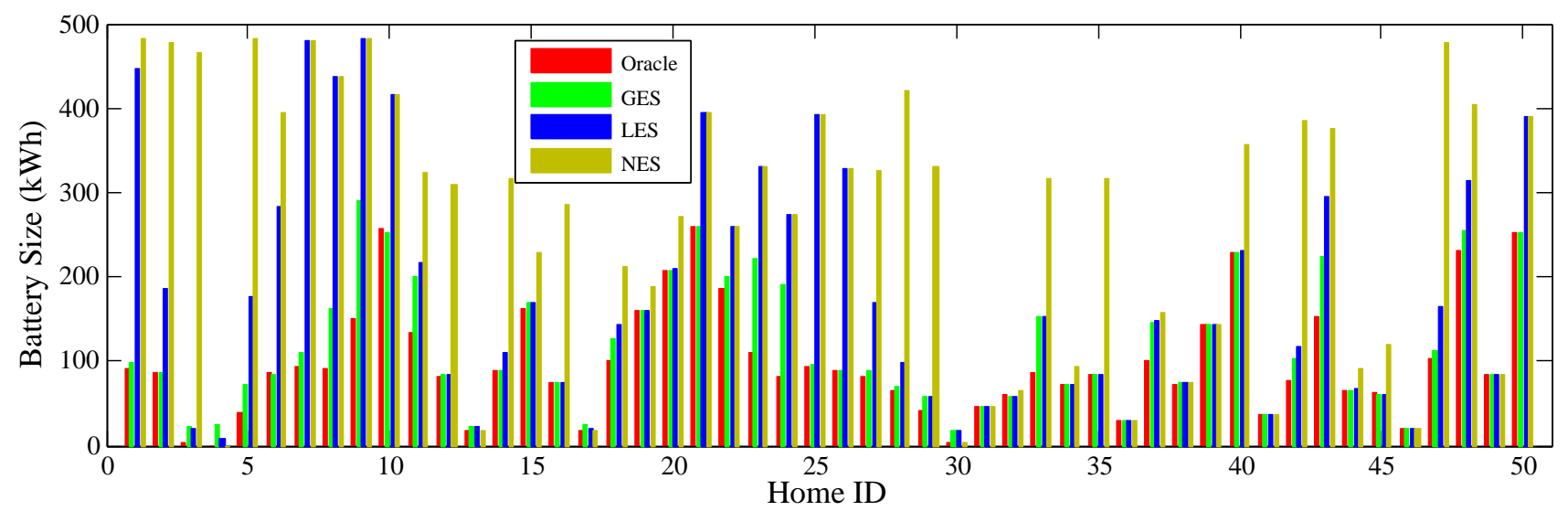

Figure 11: Battery sizes needed for homes

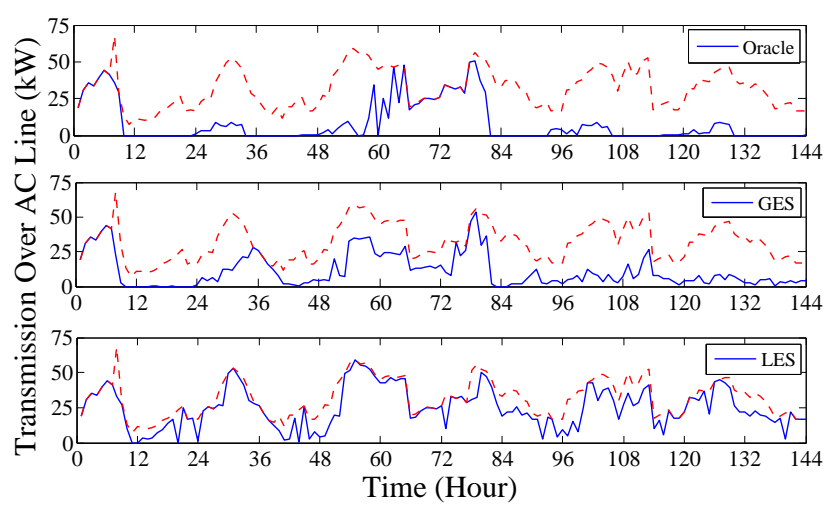

Figure 12: Energy transmission over AC line (Three algorithms (solid line) compared to NES (dash line))

day, homes do not need to get energy from AC line. LES needs less energy than NES especially when there is not enough harvesting energy at night. The peak of AC line transmission is also reduced from $70 \mathrm{kWh}$ to $50 \mathrm{kWh}$ in all three algorithms.

Figure 13 shows the energy transmission by DC per hour. The algorithms with less AC line transmission need more from DC, and NES does not have DC transmission. Note that in our algorithm, the prediction is not always accurate; thus a home may anticipate transferring more energy than it will harvest in the next window. In this case, it needs to get energy from AC line. Thus, even though energy transmission from AC line of our algorithm is larger than Oracle, DC transmission is still close to Oracle.

\subsubsection{Impact of Battery Size}

As shown before, our algorithm can reduce the battery size needed by homes compared to NES. However, in some situations, the required large battery size is still not acceptable. In this section, we investigate the impact of battery size. We set the battery size to store energy harvested in two consecutive windows and show the amount of energy transmission loss savings with different window size in Figure 14. The result is using our algorithm for 50 homes with five clusters and one cluster. With the increase of the window size, the battery size increases for both five clusters and one cluster. Energy loss savings also generally increases with window size. The reasonable explanation is that with large

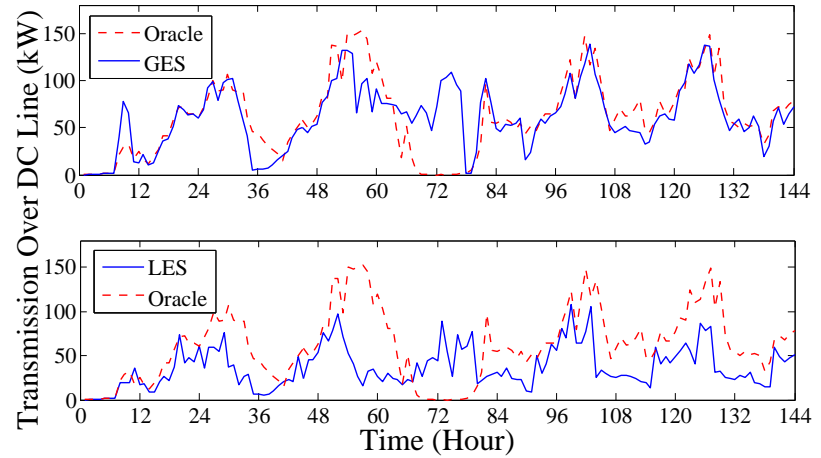

Figure 13: Energy transmission over DC line (Two algorithms compared to LES, NES does not use DC)
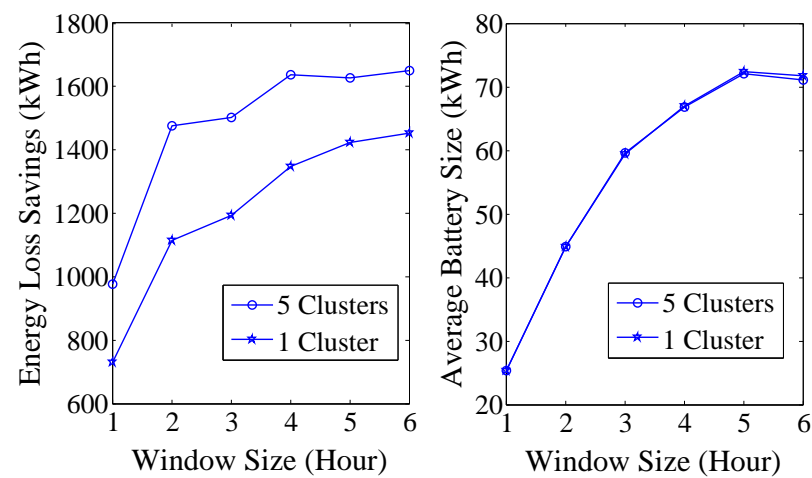

Figure 14: Energy loss savings of different battery sizes (50 homes with both five clusters and one cluster. Note that battery size for five clusters and one cluster is almost the same.)

battery size, homes can use more energy from the battery rather than relying on the $\mathrm{AC}$ line. However, the increase rate of energy loss savings decreases with the increase of window size. Thus for different situations, the tradeoff should be balanced by the price of battery and the energy loss savings.

\subsubsection{Impact of Number of Homes In One Cluster}

We show the impact of number of homes in one cluster to demonstrate the robustness of our system. Figure 15 shows the energy loss savings of different number of homes in a cluster (10 and 50). As the total number of homes is fixed 


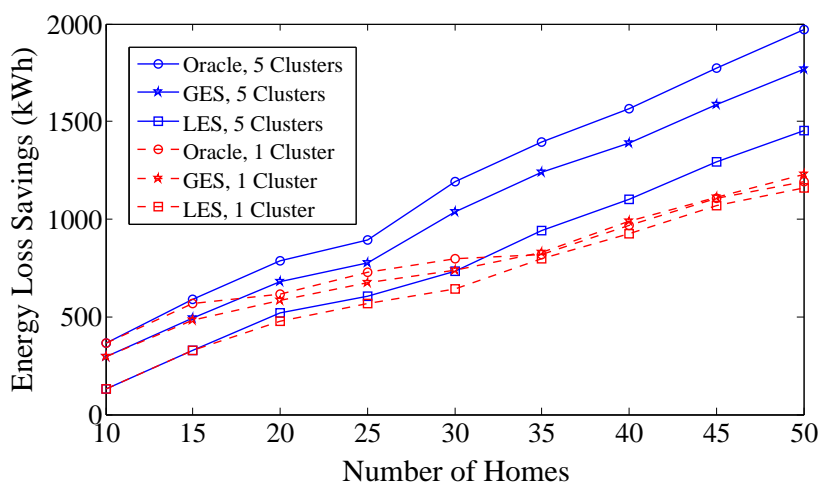

Figure 15: Energy loss savings of different number of homes in one cluster

\begin{tabular}{|c|c|c|c|c|c|c|}
\hline Window Size & 1 & 2 & 3 & 4 & 5 & 6 \\
\hline Cost $\left(\$ 10^{5}\right)$ & 3.26 & 5.21 & 6.68 & 7.41 & 7.93 & 7.83 \\
\hline Benefit $\left(\$ 10^{5} / y r\right)$ & 0.65 & 0.93 & 1.00 & 1.05 & 1.05 & 1.05 \\
\hline Years for Return & 5.01 & 5.60 & 6.68 & 7.01 & 7.53 & 7.44 \\
\hline
\end{tabular}

Table 2: Cost and Benefit for Different Window Size

at 50, and the increase of number of homes per cluster, the number of clusters decreases. Generally, the performance with five clusters is better than with only one cluster. With more clusters, the energy transmission can occur in parallel, which increases the amount of energy transmitted over the DC line in a window size, and then reduces the $\mathrm{AC}$ line energy transmission; the reduction of AC energy transmission, in turn, it reduces the total energy loss. Besides, when there is only one cluster, the performance gap between different algorithms decreases with the number of homes. This is because as the number of homes increases, the amount of energy transmission that can occur in a window size decreases. Therefore the performance of our algorithm and Oracle reduces.

\section{DISCUSSION}

Return-on-Investment. To implement energy sharing, the main expense is to construct the DC line, use solar panels and large battery to harvest and store energy. For battery, the price is around $\$ 200 / \mathrm{kWh}$. For solar panels, the price is around $\$ 0.6 /$ Watt. For wires, the maximum power of DC line is around $150 \mathrm{~kW}$, thus we choose wires with 2 AWG and the price is $\$ 14.58$ for 500 feet. Assuming the total distance of 50 homes is 10 miles, then the total price for wires is $\$ 1539$. The price of other equipment, such as inverter and energy monitor, is listed in our earlier work [18]. The benefit brought with our system design is mainly due to the savings of energy transmission over AC lines. The utility price in Amherst, MA is $\$ 0.13 / \mathrm{kWh}$. We present an analysis of benefit and cost in Table 2 . The benefit and cost both increase with the window size. However, due to the high increase ratio of battery size shown in Figure 14, the cost increases faster than benefit. To return the investment sooner, window size of one hour should be chosen for practicality.

Centralized vs. Distributed. Our current system design is a centralized control. A centralized cluster controller needs to collect energy harvesting and consumption information of all the homes in a cluster. However, since the number of homes in a cluster is limited, the computation and storage consumption is low. After the energy is shared in a cluster, the cluster controllers need only to send the energy information of homes that still have energy surplus or shortage to a higher layer controller. Thus, the total computation and storage overhead is low. Moreover, we also plan to develop distributed control in future, which allows homes to collect energy information from their neighbors to further reduce the computation and storage cost of controllers.

Algorithm under TOU (Time of Use). Nowadays, the price of electricity at different time varies under TOU. Although our solution is not based on the TOU, it can be easily revised to cope with the TOU. When the price of energy is high, we can simply assume the energy loss over AC line is larger, and when the price is low, the energy loss over $\mathrm{AC}$ line is smaller. Then with dynamic energy loss over AC line at different time, we can still have the optimum solution for all the homes with lowest cost.

Price Design. Since we are mainly concerned with minimizing the energy loss in energy sharing, we do not pay much attention to the price of energy shared between homes. However, when considering the price factor, the incentives for homes to share energy may change. Some homes may not have enough incentives to share energy if the price of shared energy is low. We plan to adapt some market-based solutions to design the price of shared energy in the future.

\section{RELATED WORK}

This work builds on our experiences gained from energy management in sensor networks projects $[28,31,30,26,32$, 29]. Our research is also related to energy efficient buildings, smart power grid, and workload scheduling.

- Research in energy efficient buildings mainly focus on energy auditing [12], design of control algorithms [10, 19] to reduce energy consumption inside a single building. Our work takes a different approach by investigating energy sharing among multiple nearby homes.

- In smart power grid, researchers have i) developed models based on measurement from phaser measurement units to solve the wide area control problem of large-scale power systems [6], [7], [8], ii) investigated the integration of renewable energy into power grid $[9$, $14,16,17,27]$, and iii) optimized the packing size of large scale batteries to improve battery utilization in microgrids [11]. Our work builds on previous works, but concentrates on minimizing on minimizing energy sharing loss over DC line at the small community level.

- Since the price of electricity varies over time, related works focus on scheduling the workload to reduce the energy cost [21], [22]. [20] utilizes both location and time diversity of electricity price under multi-regional electricity markets to minimize the total electricity cost of Internet Data Centers (IDCs). [33] takes a standard constrained model predictive control approach to smooth power demand and shave the power peak. Unlike previous works, our approach reduces the energy consumption without impacting the users' workload. 


\section{CONCLUSION}

In this paper, we addressed the mismatch between harvested and consumed energy in individual homes by proposing energy sharing among nearby homes. To ensure the efficiency of the energy sharing, we i) created a novel energy sharing system, ii) developed a greedy matching algorithm, and iii) designed a practical transmission scheduling method.

We evaluated our system using empirical traces of harvested solar energy and home energy consumption in Amherst, MA. Through extensive simulations, we verified that our system i) can achieve high energy efficiency with small battery size, ii) is robust with different number of homes in a cluster and different window size, and iii) can reduce $60 \%$ of energy loss from AC line.

\section{ACKNOWLEDGEMENTS}

Special thanks to our reviewers and our shepherd Dr. Le Xie for helpful feedback. This work was supported in part by the National Science Foundation grants CNS-1217791.

\section{REFERENCES}

[1] Electric Vehicle Battery. http://en.wikipedia.org/wiki/Electric_vehicle_battery\# cite_note-18k-23.

[2] Freeing the Grid: Best and Worst Practices in State Net Metering Policies and Interconnection Procedures. http://www.newenergychoices.org/uploads/ FreeingTheGrid2009.pdf, 2009.

[3] State of California Executive Order S-21-09. http://gov.ca.gov/executive-order/13269, 2009.

[4] Database of State Incentives for Renewables and Efficiency. http://www.dsireusa.org, 2010.

[5] SERC: State Environmental Resource Center. http://www.serconline.org/netmetering/stateactivity.html, 2011.

[6] A. Chakrabortty. Wide-Area Control of Large Power Systems Using Dynamic Clustering and TCSC-Based Redesigns. In IEEE Transctions on Smart Grid, Vol. 3, No. 3, pages 1503-1514, 2012.

[7] A. Chakrabortty and A. Salazar. Building a dynamic electro-mechanical model for the Pacific AC intertie using distributed synchrophasor measurements. In European Transactions on Electric Power: Special Issue on PMU Applications, Vol. 21, No. 4, pages 1657-1672, 2011.

[8] A. Chakrabortty, J. H. Chow and A. Salazar. A Measurement-based Framework for Dynamic Equivalencing of Power Systems using Wide-Area Phasor Measurements. In IEEE Transactions on Smart Grid, Vol. 1, No. 2, pages $68-81,2011$.

[9] A. Thatte, and L. Xie. Towards a Unified Operational Value Index of Energy Storage in Smart Grid Environment. In IEEE Transactions on Smart Grid, Vol. 3, No. 3, pages $1418-1426,2012$.

[10] M. Behl, M. Aneja, H. Jain, and R. Mangharam. Enroute: An energy router for energy-efficient buildings. In IPSN, 2011.

[11] F. J. Jin and K. G. Shin. Pack sizing and reconfiguration for management of large-scale batteries. In Proceedings of International Conference on Cyber-Physical Systems, 2012.

[12] X. Jiang, M. Van Ly, J. Taneja, P. Dutta, and D. Culler. Experiences with a high-fidelity wireless building energy auditing network. In SenSys, pages 113-126, 2009.

[13] A. Kansal, J. Hsu, S. Zahedi, and M. B. Srivastava. Power management in energy harvesting sensor networks. ACM Transction on Embedded Computer Systems, 6(4):1539-9087, 2007.

[14] L. Xie, Y. Gu, A. Eskandari, and M. Ehsani. Fast MPC-based Coordination of Wind Power and Battery
Energy Storage Systems. In Journal of Energy Engineering, Vol. 138, No. 2, pages $43-53,2012$.

[15] D. Larruskain, I. Zamora, A. MazÃşn, O. Abarrategui, and J. Monasterio. Transmission and distribution networks: Ac versus dc. 9th Spanish-Portuguese Congress on Electrical Engineering, 2005.

[16] M.D. Ilic, L. Xie and J. Joo. Efficient coordination of wind power and price-responsive demand Part I: theoretical foundations. In IEEE Transactions on Power Systems Vol. 26, No. 4, pages $1875-1884,2011$.

[17] M.D. Ilic, L. Xie and J. Joo. Efficient coordination of wind power and price-responsive demand Part II: case studies. In IEEE Transactions on Power Systems Vol. 26, No. 4, pages 1885 - 1893, 2011.

[18] A. Mishra, D. Irwin, P. Shenoy, J. Kurose, and T. Zhu. Smartcharge: Cutting the electricity bill in smart homes with energy storage. e-Energy, 2012.

[19] T. Nghiem, M. Behl, G. Pappas, and R. Mangharam. Green scheduling: Scheduling of control systems for peak power reduction. In International Green Computing Conference and Workshops (IGCC), 2011.

[20] L. Rao, X. Liu, M. Ilic, and J. Liu. Distributed coordination of internet data centers under multiregional electricity markets. Proceedings of the IEEE, 100(1):269-282, 2012.

[21] L. Rao, X. Liu, L. Xie, and W. Liu. Minimizing electricity cost: Optimization of distributed internet data centers in a multi-electricity-market environment. In INFOCOM, 2010.

[22] L. Rao, X. Liu, L. Xie, and W. Liu. Coordinated energy cost management of distributed internet data centers in smart grid. IEEE Transactions on Smart Grid, 3(1):50 -58, 2012.

[23] S. M. Schoenung. Energy storage systems cost update [a study for the doe energy storage systems program]. Tech. Rep. SAND2011-2730, Sandia National Laboratories, 2011.

[24] N. Sharma, J. Gummeson, D. Irwin, and P. Shenoy. Cloudy Computing: Leveraging Weather Forecasts in Energy Harvesting Sensor Systems. In SECON, 2010.

[25] T. Zhu, A. Mishra, D. Irwin, N. Sharma, P. Shenoy, and D. Towsley. The Case for Efficient Renewable Energy Management for Smart Homes. In ACM BuildSys, 2011.

[26] T. Zhu, A. Mohaisen, Y. Ping, and D. Towsley. DEOS: Dynamic Energy-Oriented Scheduling for Sustainable Wireless Sensor Networks. In INFOCOM, 2012.

[27] T. Zhu, S. Xiao, P. Yi, D. Towsley, and W. Gong. A Secure Energy Routing Protocol for Sharing Renewable Energy in Smart Microgrid. In IEEE SmartGridComm, 2011.

[28] T. Zhu, Y. Gu, T. He and Z.-L. Zhang. Eshare: A Capacitor-driven Energy Storage and Sharing Network for Long-term Operation. In SenSys, 2010.

[29] T. Zhu, Z. Zhong, T. He, and Z.-L. Zhang. Energy-Synchronized Computing for Sustainable Sensor Networks. In Elsevier Ad Hoc Networks Journal, 2010.

[30] T. Zhu, Z. Zhong, T. He and Z.-L. Zhang. Exploring link correlation for efficient flooding in wireless sensor networks. In Proceedings of the 7th USENIX conference on Networked Systems Design and Implementation (NSDI), 2010.

[31] T. Zhu, Z. Zhong, Y. Gu, T. He and Z.-L. Zhang. Leakage-aware Energy Synchronization for Wireless Sensor Networks. In MobiSys, 2009.

[32] T. Zhu, Z. Zhong, Y. Gu, T. He, and Z.-L. Zhang. Feedback Control-based Energy Management for Ubiquitous Sensor Networks. In IEICE Transactions on Communications, Vol. E93-B, No.11, pages 2846-2854, Nov. 2010.

[33] J. Yao, X. Liu, W. He, and A. Rahman. Dynamic control of electricity cost with power demand smoothing and peak shaving for distributed internet data centers. In $I C D C S$, 2012 . 